\title{
Update on Non-neuraxial Labor Analgesia
}

\author{
Dana Karol $^{1}$ (D) Carolyn F Weiniger ${ }^{1}$
}

Accepted: 9 June 2021 / Published online: 18 August 2021

(C) The Author(s), under exclusive licence to Springer Science+Business Media, LLC, part of Springer Nature 2021

\begin{abstract}
Purpose of Review This review will present recent updates for the use of non-neuraxial analgesia for laboring women. Recent Findings Non-neuraxial labor analgesia by nitrous oxide and intravenous opioids are described, and safety concerns with regard to remifentanil administration have been a major focus of recent studies. Fentanyl and nitrous oxide offer some efficacy, albeit less than remifentanil, however with a greater safety margin.

Summary Women may request or require alternatives to neuraxial labor analgesia, in some cases due to concurrent comorbidities. Remifentanil offers the most efficacious alternative analgesia option; however, safety concerns may preclude widespread use.
\end{abstract}

Keywords Remifentanil $\cdot$ Non-neuraxial $\cdot$ Epidural $\cdot$ Labor $\cdot$ Safety $\cdot$ Analgesia

\section{Introduction}

While labor can be a wonderful affirming process, it is painful and can be protracted and traumatic for some women. The ability to choose efficacious analgesia options is an essential contribution to the labor experience. Prior to labor, women may seek information regarding labor analgesia options and may not necessarily have a fixed decision regarding the analgesia they prefer [1-3]. Many factors may impact the decision regarding labor analgesia, including concurrent comorbidities such as high body mass index, cardiac disease, obstetric history such as prior cesarean delivery, preeclampsia, and available monitoring options. This review will discuss updates in non-neuraxial labor analgesia options over the past 5 years.

\section{Pre-admission Obstetric Anesthesiologist Consultation}

Ideally, all women could consult with an anesthesiologist regarding her optimal analgesia options prior to labor, enabling

This article is part of the Topical Collection on Obstetric Anesthesia

Carolyn F Weiniger

carolynw@tlvmc.gov.il

1 Division of Anesthesia and Critical Care and Pain, Tel Aviv Sourasky Medical Center, 6 Weizmann St, Tel Aviv, Israel selection of the most appropriate options, based on individualized information $[4 \cdot \bullet]$. A more diverse, older laboring population may have associated concurrent comorbidities such as cardiovascular conditions, thromboembolism, and cardiomyopathy which are more common causes of peripartum death [5]. Women receiving thromboprophylaxis and anticoagulation may desire neuraxial analgesia. Timing of these medications can impact the available options. Thus, concurrent comorbidities and disease severity require a flexible approach to optimal individualized labor analgesia [6].

\section{Neuraxial Analgesia}

While neuraxial analgesia provides the most effective labor pain relief, availability, contraindications, and preferences may impact its use in labor [7]. Souza et al. evaluated use of labor analgesia for vaginal delivery in Africa, Asia, Middle East, and Latin America, in a cross-sectional, facility-based survey of 359 healthcare facilities in 29 countries [8]. Sociodemographic and obstetric characteristics of laboring women who did and did not receive labor analgesia were presented. Among the 221,345 women who underwent vaginal delivery, only $4 \%$ received labor analgesia, mostly epidural. Women who received analgesia were more likely to live in countries with a high human development index (HDI). Education was associated with increased use of labor analgesia. In the United States (US), while neuraxial analgesia use is 
more prevalent $(73 \%$ of laboring women used it, among a population of $2,625,950$ ), there are state-wide differences in use, as reported by Butwick et al. [7]. The reasons for these variations could not be defined, however likely include availability of neuraxial analgesia, and quality of obstetric anesthesia care. States with a low prevalence of neuraxial analgesia have a high proportion of the population living in rural communities, and hospital closures may impact care available [9]. In Israel, labor analgesia options were evaluated in a national survey in 2005 and again in 2016. Among 25 units (100\% response rate), the median (range) epidural rate was $60 \%$ $(22-85 \%)$, a small increase in rate from 50\% (15-93\%) reported in 2005 [10].

\section{Contraindications for Neuraxial Analgesia}

Women in the peripartum period have a 4- to 5-fold increased risk of venous thromboembolism (VTE) compared with nonpregnant women [11]. Growing indications for peripartum VTE prophylaxis, including NOAC (novel oral anticoagulants), mean that more women requiring timing or stopping recommendations in the peripartum period to enable timely neuraxial analgesia [12]. Leffert et al. led a Society of Obstetric Anesthesia and Perinatology (SOAP) consensus statement, developed with corresponding obstetric anesthesia experts that aimed to provide practical and evidence-based guidance for physician anesthesiologists to manage women receiving antepartum and/or postpartum thromboprophylaxis with unfractionated heparin (UFH) or low molecular weight heparin (LMWH) [12]. Women with severe morbidity such as deep vein thrombosis, pulmonary embolus, mechanical heart valve, or arrhythmia may not be able to discontinue these medications in order to enable timely provision of neuraxial labor analgesia.

Other conditions that may preclude women from receiving optimal labor neuraxial analgesia include severe scoliosis and post-surgical spine instrumentation. Ideally all centers would offer a suitable alternative to neuraxial analgesia for laboring women; however, options may be limited by resources and by personnel. In the Israeli survey [10], fifteen (60\%) units offered nitrous oxide, four (16\%) patient-controlled intravenous fentanyl, and six units (24\%) offered patient-controlled intravenous remifentanil for non-neuraxial labor analgesia. Alternative options are not uniformly available, and lack consensus regarding their efficacy and safety

\section{Intravenous Opioid Labor Analgesia}

\section{Remifentanil}

The most efficacious non-neuraxial labor analgesia method appears to be remifentanil intravenous (IV) patient- controlled analgesia (PCA). While remifentanil IV-PCA is inferior to neuraxial analgesia for provision of labor analgesia $[13,14]$, it may provide a satisfactory level of labor analgesia.

Remifentanil is a piperidine derivative, $92 \%$ proteinbound, with a strong affinity for the $\mu$-opioid receptor and less so for the $\delta$ - and $\mathrm{K}$-receptors [15]. It is competitively antagonized by naloxone. Remifentanil has a rapid onset and rapid offset of effect, regardless of the duration of administration. The esterase-based metabolism of remifentanil makes its pharmacokinetics independent of end-organ function [16]. Most of this hydrolysis occurs in the skeletal muscle, and the total remifentanil clearance is $30-50 \%$ of cardiac output [17]. The remifentanil context sensitivity half-life (decrease of $50 \%$ of plasma concentration after halting a continuous infusion) is low and time-dependent. After a 3-h infusion of remifentanil administered to equipotent concentrations of alfentanil, there is full recovery of respiratory drive within $15 \mathrm{~min}$ for remifentanil, compared with $>45 \mathrm{~min}$ for alfentanil [16]. Given the rapid onset and offset and metabolism in mother and neonate, remifentanil has suitable pharmacokinetic properties for labor analgesia.

\section{Efficacy}

Remifentanil IV-PCA efficacy has been compared to alternative non-neuraxial analgesia such as meperidine (pethidine) and to neuraxial analgesia. The RESPITE study compared remifentanil IV-PCA to intramuscular meperidine in a nonblinded, 1:1 randomized controlled trial [11]. Upon request for labor analgesia, 400 women in established labor received systemic opioid pain relief, either remifentanil IV-PCA (bolus 40 $\mu \mathrm{g}$, lockout interval $2 \mathrm{~min}$ ) or meperidine intramuscular (IM) (100 mg, up to 4 hourly in frequency, maximum of four doses; up to $400 \mathrm{mg}$ per $24 \mathrm{~h}$ ). Although significantly fewer women randomized to receive remifentanil requested neuraxial analgesia (19 versus $41 \%$ ), $61 \%$ of all women approached refused to enroll in the study; thus, there was a strong enrollment bias against women who wished to receive neuraxial analgesia. In addition, it is unclear from this study the duration of efficacious opioid analgesia until request for neuraxial analgesia.

Most studies have not reported duration of remifentanil administration [18]. The maternal labor experience was reported using surveys and postpartum telephone interviews on women from the RESPITE study; 30 received remifentanil IV-PCA and 19 IM meperidine [1]. Some women who received remifentanil IV-PCA reported effective pain relief and ability to remain alert and focused during labor, while some reported restricted movements and a fear that the remifentanil infusion would run out.

In an observation from a single Swedish university center (2009-2016), Thorbiörnson et al. investigated duration of active labor, delivery mode, and maternal and neonatal morbidity and maternal satisfaction with remifentanil IV-PCA $(\mathrm{n}=$ 
69) versus neuraxial analgesia $(n=138)$, hypothesizing that PCA would be associated with a shorter labor duration compared to neuraxial analgesia. Women who self-selected to receive remifentanil IV-PCA had shorter active labor duration $5.6 \pm 3.3 \mathrm{~h}$ compared to $8.5 \pm 4.4 \mathrm{~h}$ for neuraxial (epidural) analgesia, $\mathrm{P}=0.001$, and higher rate of spontaneous delivery. Interestingly, women levels of satisfaction with the labor analgesia were similar for both remifentanil IV-PCA and neuraxial analgesia. In this study, there were similar rates of maternal $\left(\mathrm{SpO}_{2}<95 \%\right.$, sedation) and neonatal (Apgar $<7$ at 5 -min, neonatal intensive care unit admission, sedation) morbidity [19].

Melber et al. summarized data from an ongoing self-report system from 31 European centers where women receive remifentanil IV-PCA $[20 \bullet$. This database is continually updated, available as the remiPCA network-https://www. remipca.org/php/en/index.php (Figure 1). The network was established to set worldwide standards and to monitor maternal and neonatal outcomes when using remifentanil IV-PCA during labor. The data collected include effectiveness, and severe adverse effects such as need for ventilation and maternal or neonatal cardiopulmonary resuscitation. A summary publication data from 2010 to 2015 reported that among 5740 data, no women required ventilation or cardiopulmonary resuscitation, yet $25 \%$ of women had hypoxia (oxygen saturation $<94 \%$ ). Neonatal cardiopulmonary resuscitation, potentially related to remifentanil, occurred in $0.3 \%$, and the rate of neonatal supplemental oxygen was $5 \%$. Melber et al. reported that there were no complications in the centers that used remifentanil IV-PCA at least 10 times in the past year, suggesting that experience matters $[20 \bullet \bullet]$.

Murray et al. report data from Northern Ireland Maternity System (NIMATS), where remifentanil IV-PCA is a freely available option for women who prefer this instead of neuraxial analgesia. Data were retrospectively retrieved from all obstetric units in Northern Ireland for women who received remifentanil IV-PCA, diamorphine IM, or neuraxial analgesia during labor between 2005 and 2014. Throughout the 10-year period, remifentanil IV-PCA was the most popular form of labor analgesia, selected by $32 \%(8170 / 25617)$ of women. Women who selected remifentanil IV-PCA had similar rates of instrumental and operative delivery, and neonatal Apgar scores. Neonatal resuscitation and neonatal intensive care unit admission were not more likely among women choosing remifentanil IV-PCA. Women were monitored with the constant presence of a midwife, which enables a prompt response to clinically significant event such as hypoventilation. Interestingly, there was a low conversion rate to neuraxial analgesia, rising from 8 to $9 \%$ during the 10 -year period [21].

\section{Remifentanil Safety}

While remifentanil IV-PCA appears to provide efficacious labor analgesia, there are potential side effects. These include maternal hypoxia $[13,14]$, respiratory and cardiac arrest $[22 \cdot \bullet$, 23], pruritus, and/or nausea and vomiting [13]. Laboring women who received remifentanil IV-PCA require a suitable monitor to alert for apnea [13]. Stocki et al. conducted a single-center non-blinded randomized controlled trial among healthy women who had a single fetus and vertex presentation. Women received either remifentanil IV-PCA, titrated from $20 \mu \mathrm{g}$ up to a maximum bolus dose of $60 \mu \mathrm{g}$ (lockout interval of 1 to $2 \mathrm{~min}$ ), or neuraxial analgesia with PCEA $0.1 \%$ bupivacaine with $2 \mu \mathrm{g} / \mathrm{mL}$ fentanyl (initiation bolus 15 $\mathrm{mL}$; maintenance bolus $10 \mathrm{~mL}$, lockout interval $20 \mathrm{~min}$, basal infusion $5 \mathrm{~mL} / \mathrm{h}$ ). The primary outcome was efficacy, and the secondary outcome was safety [13]. Supplementary oxygen was administered continuously during the respiratory monitoring period. Among 40 women, $\mathrm{n}=19$ randomized to receive remifentanil, $\mathrm{n}=20$ randomized to receive LEA; mean
Figure 1 Data from the RemiSafe network, illustrating increased reporting of cases using intravenous remifentanil patientcontrolled analgesia for labor. These data are continually updated and available online at https://www.remipca.org/php/en/ index.php\#information. Permission granted for reproduction by Dr. Andrea Melber

\section{Documented RemiPCA Applications cumulated}

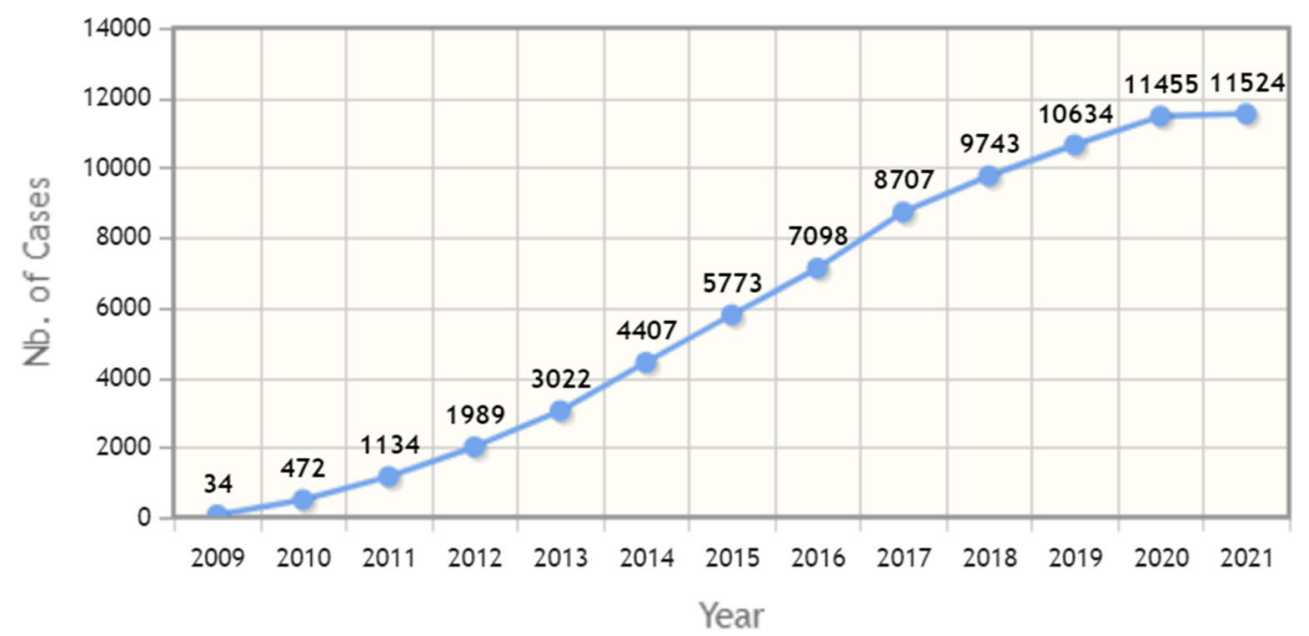


respiratory rate and $\mathrm{SpO}_{2}$ were lower in the remifentanil group, $18 \pm 4$ versus $21 \pm 4$ breaths $/ \mathrm{min}$ and $96.8 \% \pm 1.4$ versus $98.4 \pm 1.2$, respectively. There were 9 apnea events; all occurred in 5/19 (26.3\%) women receiving remifentanil; Apgar scores and neonatal respiratory outcomes were similar [22••]. The study authors concluded that while remifentanil has some efficacy, women should be monitored by someone able to identify and manage apnea that does occur. Logtenberg et al. reported a Dutch national survey to identify severe adverse effects when remifentanil IV-PCA was used in labor. Sixty-one centers participated, reporting 17 cases of maternal desaturation; 10 maternal cases of apnea, bradycardia, and/or cardiac arrest; and two neonatal cases of respiratory depression, over a period of more than 10 years of remifentanil IVPCA use. All serious adverse events were resolved without irreversible damage.

Tveit et al. performed a prospective, observational study using stepwise bolus doses of on remifentanil IV-PCA, during the first and second stages of labor. Laboring women with normal term, singleton pregnancies were recruited. Safety outcomes measured included respiratory rate, oxygen saturation, and maternal sedation, recorded every $15 \mathrm{~min}$. The nadir oxygen saturation was $91 \%$, and respiratory rate was 9 breaths/ min; $11(27 \%)$ women received supplemental oxygen due to oxygen saturations $<92 \%$. As with previous studies, the authors concluded that maternal sedation and respiratory depression occur when using remifentanil IV-PCA, and that maternal monitoring is mandatory, yet neonatal data were reassuring [14].

While the recommendation of 1:1 nursing increases the chance of identifying respiratory depression [18], this may not be universally available. The difficulty to provide suitable staff monitoring is further compounded in the setting of increasing numbers of deliveries and insufficient concurrent staff increases [10]. Respiratory monitoring, including capnography, cannot be viewed as an alternative to the presence of a trained clinician. Such issues currently limit the widespread adoption of remifentanil IV-PCA as a routine choice for labor analgesia [10]; thus, other alternative analgesic options are required.

\section{Fentanyl}

Fentanyl is an opioid, narcotic drug usually administered for perioperative analgesia or to manage severe pain. Fentanyl has a high context sensitivity half-life; thus, after continuous infusion over $2 \mathrm{~h}$, when halted, it takes at least $50 \mathrm{~min}$ to achieve $50 \%$ decrease in plasma concentration. For this reason, most labor analgesia studies halted fentanyl before second stage of labor, for neonatal safety.

Marwah et al. performed a retrospective cohort study (2005-2010) on laboring women (> 24 weeks' gestational age) to compare the analgesic efficacy and adverse effects on neonatal outcome of administering remifentanil $(n=47)$ versus fentanyl $(\mathrm{n}=51)$ IV-PCA. Women who received neuraxial analgesia were excluded. The remifentanil protocol comprised a PCA bolus $0.25 \mu \mathrm{g} / \mathrm{kg}$ with a lockout interval of $2 \mathrm{~min}$, a 4-h limit of $3 \mathrm{mg}$, and a background infusion of $0.025-0.05 \mu \mathrm{g} / \mathrm{kg} / \mathrm{min}$, and the fentanyl regime comprised a PCA bolus 25-50 mic with a lockout interval of 3 to 6 min and a 4-h limit of 1-1.5 mg. Outcomes reported included maternal hourly pain scores, sedation scores, adverse effects, and neonatal outcomes. There was no significant difference in pain scores (recorded on a verbal pain score scale $0-10$ ), between the two groups, a slight decrease from baseline. There was no difference in maternal side effects between the two groups, although transient desaturation was more significantly observed when remifentanil versus fentanyl was administered ( $13 \%$ versus $2 \%$, respectively). A larger number of neonates, $59 \%$, required resuscitation (bag mask/ventilation-intubation) after the mother received fentanyl, compared with $25 \%$ after remifentanil [24].

In 2012, Hosokawa et al. investigated the safety of fentanyl IV-PCA during labor in a single-center retrospective study of fentanyl use $(n=129)$ versus no analgesia $(n=697)$, after excluding women who underwent cesarean delivery from the retrospective database. Women were $\geq 35$ weeks' gestational age, and data were collected from a 3-year period (2005-2007). The fentanyl IV-PCA was administered with a loading dose of $0.05 \mathrm{mg}$ fentanyl, followed by bolus injection of $0.02 \mathrm{mg}$ fentanyl, lockout interval of $5 \mathrm{~min}$, that was initiated at the parturient's request and discontinued before the second stage of labor. During labor, both maternal and fetal heart rates, oxygen saturation as measured by pulse oximetry $\left(\mathrm{SpO}_{2}\right)$, respiratory rate, sedation and nausea scores, and neonatal outcomes (umbilical arterial pH, Apgar scores) were recorded. Use of fentanyl IV-PCA was associated with a prolonged duration of labor and increased the need for instrument-assisted delivery and oxytocin use. There were no obvious maternal or neonatal complications of fentanyl use that were recorded. Although a significantly lower rate of emergency cesarean delivery was noted, there were unmeasured confounders associated with this finding, and the authors noted the possible effect of using IV-PCA on the rate of maternal request for cesarean delivery [25].

In summary, fentanyl IV-PCA is a possible analgesia option when neuraxial analgesia is contraindicated. Nevertheless, fentanyl IV-PCA can cause neonatal side effects; thus, naloxone should be available on the labor ward and a pediatrician should be available at the delivery.

\section{Nitrous Oxide}

Nitrous oxide is an anesthetic gas that affects the $N$-methyl-Daspartate (NMDA) receptor - a glutamate receptor and ion channel protein found in nerve cells. It has antagonistic 
properties and offers rapid-onset inhaled analgesia. Zandaro et al. performed a case-control study on 62 women who received nitrous oxide versus 124 who did not. Outcomes included intensity of nitrous oxide labor pain relief, satisfaction memory by a retrospective visual analog scale (VAS, $0-10$ Numeric Rating Scale), and depression symptoms (measured by the Edinburgh Postnatal Depression Scale (EPDS)). Nitrous oxide use was associated with a lasting positive labor pain relief experience (VAS, 7.3 \pm 2.2 ) in $84 \%$ of women, and labor satisfaction memory (VAS, $8.9 \pm 1.8$ ) in $90 \%$ of women, yet did not influence EPDS subscales upon discharge [26]. The memory of labor pain was measured by asking women to rate (VAS $0-10$ ) whether they would use nitrous oxide in a subsequent delivery.

Sutton et al. retrospectively examined characteristics of 146 laboring women who used nitrous oxide (3\% of the laboring population), to identify prediction criteria for neuraxial analgesia conversion. Most women who used nitrous oxide were nulliparous (72\%), and women who had expressed an initial preference for "non-medical birth (52\%)." The conversion rate to neuraxial analgesia was $63 \%$, compared to $85 \%$ among women who did not use nitrous oxide. Only a small number of women used nitrous oxide during labor, analgesia was minimal, and most converted to neuraxial analgesia [27].

\section{Knowledge Is Power}

Disseminating the correct information to pregnant women is important to enable timely and appropriate decisions about labor analgesia. There are many varied sources of information including antenatal courses, and Internet platforms. Sutton et al. reported labor epidural searches using Google Trends for US data (2004 to 2015). The Search terms with the most significant growth were "birth with epidural," "pain after epidural," "labor without epidural," "epidural birth video," and "epidural versus natural." Searches that were increasing rapidly were epidural side effects, risks, and pain on insertion [2]. D'Souza et al. evaluated YouTube videos about labor epidural analgesia (using search key words including epidural, epidural analgesia, epidural for pregnancy, epidural experience, epidural risks). The video quality was evaluated using a modified DISCERN score, a five-question validated instrument that assesses quality of health information. Videos had over 16 million views, and 13/60 (22\%) included non-factual information about epidural analgesia [28]. Importantly, videos from medical sources had a higher mDISCERN score compared with videos by non-medical sources $(\mathrm{P}<0.001)$. Thus, while YouTube is an accessible platform for pregnant women to find information about epidural analgesia, non-factual information and low video quality are a problem. The Obstetric Anaesthetist's Association and SOAP have online resources available including alternative analgesia methods $[29,30]$. For women who may be unable to, or desire to, avoid neuraxial analgesia for labor, accurate information about alternative options should be available.

The impact of suitable analgesia selection goes beyond pain relief. Fear of pain during childbirth may be a significant concern $[22 \bullet \cdot]$. Fear of childbirth (FOC) is a subjective experience that labor and delivery can be frightening [31], leading to increased use of neuraxial analgesia, despite similar pain levels to women without FOC. In a study of 143 postpartum women queried about prior trauma, FOC was most common among women who had reported their index trauma was previous labor and delivery [32]. Fear of childbirth was investigated as a secondary analysis in a study where 409 women were randomized to receive remifentanil versus epidural $[22 \bullet \bullet]$. Those randomized to receive neuraxial analgesia reported FOC more frequently versus women who did not receive neuraxial analgesia, adjusted odds ratio $3.5,95 \%$; confidence interval (CI) 1.5-8.2. However, the frequency of FOC was not different comparing women who received remifentanil versus woman without analgesia.

During the COVID-19 pandemic, since early 2020, obstetric anesthesia management has been extremely challenging $[33,34]$. The importance of neuraxial anesthesia was highlighted as a way to avoid general anesthesia, given not just risks of aspiration and difficult intubation management for the parturient, but also risks for health care workers managing intubation with potential aerosol spread of the virus [35]. Another important consideration during Sars-CoV-2 infection is the severe inflammatory response in pregnant women [36], necessitating anticoagulation for VTE prophylaxis for pregnant women with Sars-CoV-2 infection as they are at increased risk [36]. This leads, as discussed previously, to the concern of timely cessation of anticoagulants to enable neuraxial analgesia, and the possibility that alternative analgesic measures may be required. Nitrous oxide is contentious with regard to safety during the pandemic, and some centers recommended not allowing women with active or recent SarsCoV-2 to receive nitrous oxide in labor [37].

\section{Conclusions}

Women may request or require alternative labor analgesia, for example due to concurrent comorbidities. Although women can easily access information regarding labor analgesia options, they may not be aware of alternative options, and these are usually only offered to women who cannot receive neuraxial analgesia. Remifentanil appears to offer the best non-neuraxial analgesia; however, safety concerns preclude widespread use, and maternal morbidity, including cardiac arrest, has been reported with its use in labor. Other alternatives such as fentanyl and nitrous oxide offer some efficacy, albeit less than remifentanil, however with a greater safety margin. 


\section{Compliance with Ethical Standards}

Conflict of Interest The authors do not have any potential conflicts of interest to disclose.

Human and Animal Rights and Informed Consent This article does not contain any studies with human or animal subjects performed by any of the authors.

\section{References}

Papers of particular interest, published recently, have been highlighted as:

•- Of major importance

1. Moran VH, Thomson G, Cook J, Storey H, Beeson L, MacArthur $\mathrm{C}$, et al. Qualitative exploration of women's experiences of intramuscular pethidine or remifentanil patient-controlled analgesia for labour pain. BMJ open. 2019;9(12):401-8.

2. Sutton CD, Carvalho B. What's trending now? An analysis of trends in internet searches for labor epidurals. International journal of obstetric anesthesia. 2017;30:52-7.

3. Souza MAC, Guida JG, Souza JP, Gulmezoglu JP, Betran AM, A P. WHO-MCS study group: Analgesia for vaginal birth: secondary analysis from the WHO Multicountry Survey on Maternal and Newborn Health. International Journal of Gynecology \& Obstetrics. 2021;152(3):401-8.

4.• SLM L, Vink ML, Godfried MB, Beenakkers ICM, Schellevis FG, Mol BW, et al. Serious adverse events attributed to remifentanil patient-controlled analgesia during labor in The Netherlands. International journal of obstetric anesthesia. 2019;39:22-8 This article presents a Dutch nationwide survey of adverse events when using remifentanil and provides safety recommendations.

5. Creanga AA, Syverson C, Seed K, Callaghan W. M: Pregnancyrelated mortality in the United States, 2011-2013. Obstetrics and Gynecology. 2017;130(2):366-73.

6. Moaveni DM, Cohn JH, Zahid ZD, Ranasinghe JS. Obstetric anesthesiologists as perioperative physicians: improving peripartum care and patient safety. Current Anesthesiology Reports. 2015;5(1):65-73.

7. Butwick AJ, Bentley J, Wong CA, Snowden JM, Sun E, Guo N. United States state-level variation in the use of neuraxial analgesia during labor for pregnant women. JAMA network open. 2018;1(8): e186567.

8. Souza MA, Cecatti JG, Guida JP, Souza JP, Gulmezoglu AM, Betran AP, et al. Analgesia for vaginal birth: secondary analysis from the WHO Multicountry Survey on Maternal and Newborn Health. International Journal of Gynecology \& Obstetrics. 2021;152(3):401-8.

9. Kozhimannil KB, Interrante JD, Tuttle MK, Henning-Smith C. Changes in hospital-based obstetric services in rural US counties. Jama 2020. 2014-2018;324(2):197-9.

10. Shatalin D, Weiniger C. F, Buchman, I, Ginosar, Y, Orbach-Zinger S, \& Ioscovich, A: A 10-year update: national survey questionnaire of obstetric anesthesia units in Israel. International journal of obstetric anesthesia 2019; 38: 83-92.

11. Gynecologists. ACoOa: ACOG practice bulletin no. 196: thromboembolism in pregnancy. Obstetrics and gynecology. 2018;132(1): e1-e17.

12. Leffert L, Butwick A, Carvalho B, Arendt K, Bates SM, Friedman A, et al. The Society for Obstetric Anesthesia and Perinatology Consensus Statement on the anesthetic management of pregnant and postpartum women receiving thromboprophylaxis or higher dose anticoagulants. Anesthesia \& Analgesia. 2018;126(3):928-44.

13. Stocki D, Matot I, Einav S, Eventov-Friedman S, Ginosar Y, Weiniger CF. A randomized controlled trial of the efficacy and respiratory effects of patient-controlled intravenous remifentanil analgesia and patient-controlled epidural analgesia in laboring women. Anesthesia \& Analgesia. 2014;118(3):589-97.

14. Tveit TO, Halvorsen A, Seiler S, Rosland JH. Efficacy and side effects of intravenous remifentanil patient-controlled analgesia used in a stepwise approach for labour: an observational study. International journal of obstetric anesthesia. 2013;22(1):19-25.

15. James MK, Vuong A, Grizzle MK, Schuster SV, Shaffer JE. Hemodynamic effects of GI 87084B, an ultra-short acting muopioid analgesic, in anesthetized dogs. Journal of Pharmacology and Experimental Therapeutics. 1992;263(1):84-91.

16. Glass PS, Gan TJ, Howell S. A review of the pharmacokinetics and pharmacodynamics of remifentanil. Anesthesia \& Analgesia. 1999;89(4S):7.

17. Rosow CE. An overview of remifentanil. Anesthesia \& Analgesia. 1999;89(4S):1.

18. Ronel I, Weiniger CF. Non-regional analgesia for labour: remifentanil in obstetrics. BJA education. 2019;19(11):357-61.

19. Thorbiörnson A, da Silva CP, Gupta A, Stjernholm YV. Duration of labor, delivery mode and maternal and neonatal morbidity after remifentanil patient-controlled analgesia compared with epidural analgesia. European journal of obstetrics \& gynecology and reproductive biology. 2020;X(6):100106.

20.• Melber AA, Jelting Y, Huber M, Keller D, Dullenkopf A, Girard T, et al. Remifentanil patient-controlled analgesia in labour: six-year audit of outcome data of the RemiPCA SAFE Network (2010 2015). International journal of obstetric anesthesia. 2019;39:12-21 The RemiPCA SAFE Network data shows that remifentanil patient-controlled analgesia can be applied safely. The aims of the analysis were to describe the development of the network and to report maternal and neonatal outcome data, including severe adverse events. These data are continually updated.

21. Murray H, Hodgkinson P, Hughes D. Remifentanil patientcontrolled intravenous analgesia during labour: a retrospective observational study of 10 years' experience. International journal of obstetric anesthesia. 2019;39:29-34.

$22 . \bullet$ Logtenberg SL, Verhoeven CJ, Rengerink KO, Sluijs AM, Freeman LM, Schellevis FG, et al. Pharmacological pain relief and fear of childbirth in low risk women; secondary analysis of the RAVEL study. BMC pregnancy and childbirth. 2018;18(1): 1-9 This study aimed to assess fear of childbirth and pain tolerance during labor. The authors reported that request for labor analgesia was associated with more frequent occurrence of fear of childbirth. However, women with fear of childbirth did not necessarily select epidural analgesia when remifentanil was also available, suggesting mechanisms other than pain relief are relevant.

23. Aaronson J, Abramovitz S, Smiley R, Tangel V, Landau R. A survey of intravenous remifentanil use for labor analgesia at academic medical centers in the United States. Anesthesia \& Analgesia. 2017;124(4):1208-10.

24. Marwah R, Hassan S, Carvalho JC, Balki M. Remifentanil versus fentanyl for intravenous patient-controlled labour analgesia: an observational study. Canadian Journal of Anesthesia/Journal canadien d'anesthésie. 2012;59(3):246-54.

25. Hosokawa Y, Morisaki H, Nakatsuka I, Hashiguchi S, Miyakoshi $\mathrm{K}$, Tanaka M, et al. Retrospective evaluation of intravenous fentanyl patient-controlled analgesia during labor. Journal of anesthesia. 2012;26(2):219-24.

26. Zanardo V, Volpe F, Parotto M, Giiberti L, Selmin A, Straface G. Nitrous oxide labor analgesia and pain relief memory in 
breastfeeding women. The Journal of Maternal-Fetal \& Neonatal Medicine. 2018;31(24):3243-8.

27. Sutton CD, Butwick AJ, Riley ET, Carvalho B. Nitrous oxide for labor analgesia: utilization and predictors of conversion to neuraxial analgesia. Journal of clinical anesthesia. 2017;40:40.

28. D'Souza RS, D'Souza S, Sharpe EE. YouTube as a source of medical information about epidural analgesia for labor pain. International Journal of Obstetric Anesthesia. 2020;45:133-7.

29. Pain relief in labour: How do the options compare? https://www. labourpains.com/assets/_managed/cms/files/InfoforMothers/Pain\% $20 \mathrm{Relief} \% 20 \mathrm{Comparison} \% 20 \mathrm{Card} / \mathrm{pain} \% 20 \mathrm{relief} \%$ 20comparison\%20card\%20september\%202014.pdf. Accessed 4th May 2021.

30. Information for mothers: keeping you and your baby safe and comfortable before during and after delivery. https://www.soap.org/ information-for-mothers. Accessed 4th May 2021

31. Wijma KWB, Zar M. Psychometric aspects of the W-DEQ: a new questionnaire for the measurement of fear of childbirth. Journal of Psychosomatic Obstetrics and Gynaecology. 1998;19(2):84-97.

32. Freedman SARS, Weiniger CF. Post-traumatic stress disorder and postpartum depression and their reported association with recent labor and delivery: a questionnaire survey cohort. Int J Obstet Anesth. 2020;43:18-24.
33. Breslin N, Baptiste C, Miller R, Fuchs K, Goffman D, GyamfiBannerman C, et al. Coronavirus disease 2019 in pregnancy: early lessons. American journal of obstetrics \& gynecology MFM. 2020;2(2):100111.

34. Morau E, Bouvet L, Keita H, Vial F, Bonnet MP, Bonnin M, et al. Anaesthesia and intensive care in obstetrics during the COVID-19 pandemic. Anaesthesia, critical care \& pain medicine. 2020;39(3): 345-9.

35. Zhong Q, Liu YY, Luo Q, Zou YF, Jiang HX, Li H, et al. Spinal anaesthesia for patients with coronavirus disease and possible transmission rates in anaesthetists: retrospective, single-centre, observational cohort study. British journal of Anaesthesia 2020. 2019;124(6):670-5.

36. Benhamou D, Keita H, Ducloy-Bouthors AS, CARO Working Group. Coagulation changes and thromboembolic risk in COVID-19 obstetric patients. Anaesthesia, Critical Care \& Pain Medicine. 2020;39(3):351.

37. Bauer M, Bernstein K, Dinges E, Delgado C, El-Sharawi, N, Sultan P, \& Landau R: Obstetric anesthesia during the coronavirus disease 2019 pandemic. Anesthesia and Analgesia 2020

Publisher's Note Springer Nature remains neutral with regard to jurisdictional claims in published maps and institutional affiliations. 